\title{
Ultra-long-haul transmission of 7×42.9Gbit/s PS-QPSK and PM-BPSK
}

\author{
Carsten Behrens, Domaniç Lavery, David S. Millar, Sergejs Makovejs, Benn C. Thomsen, Robert I. Killey, \\ Seb J. Savory and Polina Bayvel \\ Optical Networks Group, Dept. of Electronic \& Electrical Engineering, University College London, UK \\ Authore-mail address: c.behrens@ee.ucl.ac.uk
}

\begin{abstract}
We report on ultra-long haul transmission of polarization-switched QPSK (PS-QPSK) and polarization-multiplexed BPSK (PM-BPSK) at 42.9Gbit/s per WDM channel. Although achieving similar transmission distances in excess of $13,600 \mathrm{~km}$, PS-QPSK offers a significant reduction in receiver complexity due to a lower symbol-rate.

OCIS codes: (060.2330) Fiber optics communication; (060.4080) Modulation.
\end{abstract}

\section{Introduction}

To date, transmission over ultra-long haul transmission links has been carried out using conventional polarizationmultiplexed signal formats, e.g. polarization-multiplexed binary phase shift keying (PM-BPSK) [1] and quadrature phase shift keying (PM-QPSK) [2]. However, these modulation formats are tailored to a 2-dimensional channel with circularly-symmetric Gaussian noise despite the fact that an optical wave offers 4 degrees of freedom (2 quadratures in 2 polarizations). Recently, work to determine the optimum modulation format for this higher dimensional channel by solving a 4-dimensional sphere packing problem has been carried out[3, 4]. In this work, polarization-switched QPSK has been proposed as the modulation format with the highest asymptotic power efficiency and an asymptotic sensitivity gain of $1.76 \mathrm{~dB}$ over BPSK, which is currently the only commercially available option for transpacific transmission at $40 \mathrm{~Gb} / \mathrm{s}[5]$.

In this paper we present, for the first time, a comparison of PS-QPSK and PM-BPSK on an ultra-long-haul transmission link, extending the recent work experimentally demonstrating PS-QPSK and its comparison with PMQPSK [6]. Wavelength-division-multiplexed (WDM) transmission is carried out on a $50 \mathrm{GHz}$ grid with a linerate of 42.9Gbit/s per WDM channel demonstrating similar transmission distances for both modulation formats. However, due to its lower symbol-rate PS-QPSK offers significantly lower DSP complexity by allowing for a more than 50\% reduction in length of the FIR-filter for chromatic dispersion compensation.

\section{Experimental Setup}

To compare the transmission performance of PM-BPSK and PS-QPSK we used an external cavity laser (ECL) at $1553 \mathrm{~nm}$ with a linewidth of $100 \mathrm{kHz}$ surrounded by 6 DFB-lasers on $50 \mathrm{GHz}$ frequency grid. BPSK was generated by driving two arms of an IQ-modulator with $21.45 \mathrm{Gbit} / \mathrm{s} 2^{15}-1$ long pseudo-random binary sequences (PRBS) using DATA and $\overline{\mathrm{DATA}}$ outputs of a pulse pattern generator. The IQ-modulator was followed by a polarization multiplexing stage with a relative delay of 48 symbols yielding PM-BPSK (Figure 1 (a)). In the case of PS-QPSK, the IQ-modulator was driven with two decorrelated 14.3Gbit/s PRBS-15 patterns to obtain QPSK, which was

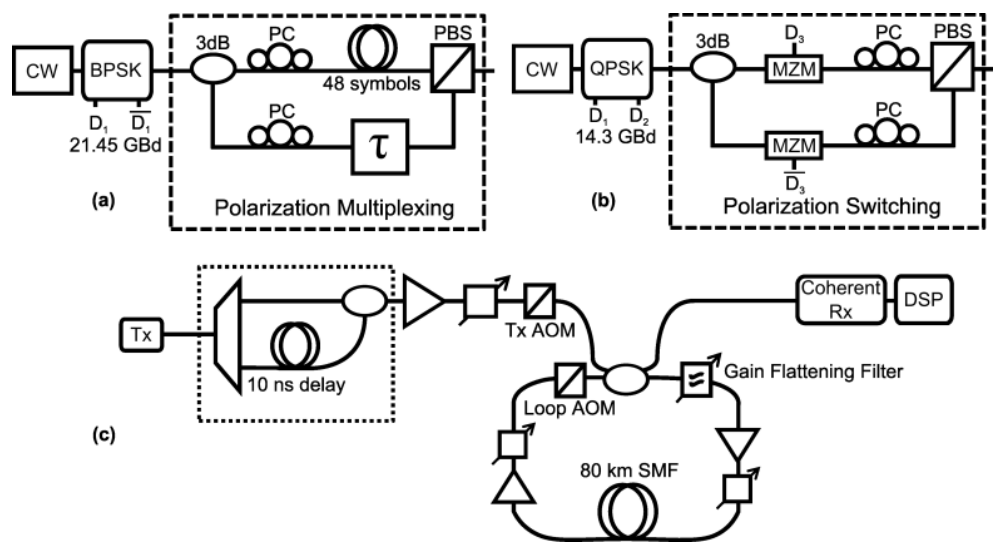

Figure 1: Schematic of (a) the PM-BPSK transmitter, (b) the PS-QPSK transmitter, and (c) the single span recirculating loop with coherent receiver used to investigate transmission performance. 
followed by a polarization switching stage consisting of two parallel Mach-Zehnder modulators (MZMs) (Figure 1 (b)). The MZMs were driven with inverse data patterns to block one or the other polarization to generate the PSQPSK. Even and odd channels were subsequently separated with a $50 \mathrm{GHz}$ interleaver and recombined with a relative delay of $10 \mathrm{~ns}$ to decorrelate neighbouring channels. The WDM-signal was then launched into a single-span recirculating loop with $80.24 \mathrm{~km}$ of standard single mode fiber (SMF). The accumulated chromatic dispersion per recirculation was $1347 \mathrm{ps} / \mathrm{nm}$ and the span loss was $15.4 \mathrm{~dB}$ [7]. Two EDFAs with noise figures of $4.5 \mathrm{~dB}$ and a fixed output power of $17 \mathrm{dBm}$ were used to amplify the signal while variable optical attenuators were used to set the launch power and balance the loop. A Mach-Zehnder filter was used to equalize the wavelength-dependent gain of the EDFAs.

The signal was detected with a phase and polarization-diverse coherent receiver using a pair of balanced PINs to receive each quadrature. The local oscillator was an ECL with $100 \mathrm{kHz}$ linewidth whose frequency was tuned to ensure that the frequency offset did not exceed $1 \mathrm{GHz}$. The signal was digitized with a digital sampling oscilloscope (DSO) with an electrical bandwidth of $16 \mathrm{GHz}$ and processed offline. After the signal had been deskewed, normalized and resampled, chromatic dispersion was compensated digitally. For PS-QPSK, a polarization-switched constant modulus algorithm (PS-CMA) equalizer with least-mean squares (LMS) updating followed by a modified Viterbi \& Viterbi phase recovery was used [7, 8]. In the case of PM-BPSK, joint equalization and phase-recovery was performed in a similar manner to that described in [9] for PM-QPSK.

\section{Transmission results at $\mathbf{4 2 . 9 G b i t / s}$}

Figure 2 shows back-to-back measurements of the receiver sensitivity for (a) PM-BPSK and (b) PS-QPSK. The results are plotted on a double-log scale and fitted linearly to ease comparison between the formats as well as against theoretical sensitivity limits. Single channel PM-BPSK shows an implementation penalty of $0.4 \mathrm{~dB}$ at $\mathrm{BER}=3.8 \times 10^{-3}$ as opposed to $0.8 \mathrm{~dB}$ for PS-QPSK. Adding more WDM channels to the signal resulted in an additional penalty of $0.2 \mathrm{~dB}$ in both cases, which is due to coherent crosstalk induced by neighbouring channels. Taking this into account it is worth noting that, at a BER of $3.8 \times 10^{-3}$, PS-QPSK preserves $0.4 \mathrm{~dB}$ of the theoretical sensitivity advantage of $0.75 \mathrm{~dB}$ over PM-BPSK.

(a)

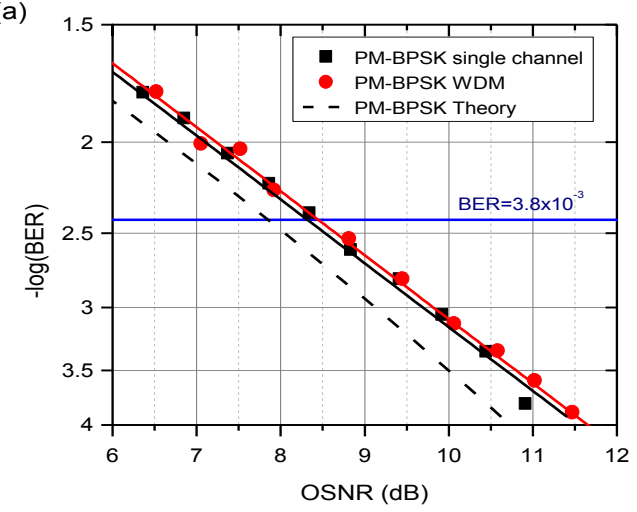

(b)

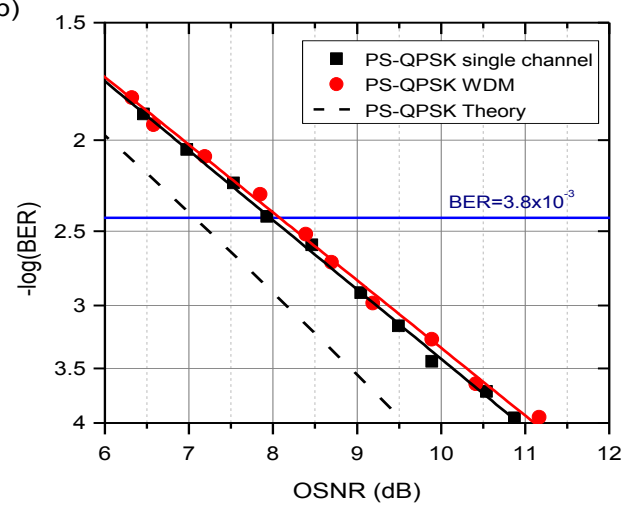

Figure 2: Back to back measurement of OSNR sensitivity for single channel and WDM configuration of (a) PM-BPSK and (b) PS-QPSK

To characterize the transmission performance of both modulation formats, 7 WDM channels were launched into a recirculating loop and the launch power per channel was varied between -14 and $4 \mathrm{dBm}$ to determine the maximum transmission distance at $\mathrm{BER}=3.8 \times 10^{-3}$. Figure 3 shows the experimental results which have been fitted with a polynomial fit [10] to emphasize the trend in the linear and nonlinear transmission regimes.

The improved sensitivity of PS-QPSK with respect to PM-BPSK observed in the back-to-back measurements translates directly into a $0.4 \mathrm{~dB}$ improvement in the linear region of the reach curve. However, on the nonlinear part of the reach curve, PM-BPSK is up to $2 \mathrm{~dB}$ more resilient towards nonlinearities than PS-QPSK, which we attribute to a higher phase-margin between adjacent constellation points. The higher susceptibility of PS-QPSK to nonlinear distortions leads to a $1 \mathrm{~dB}$ lower optimum launch power of $-3.5 \mathrm{dBm}$ compared to $-2.5 \mathrm{dBm}$ for PM-BPSK.

However, Figure 3 indicates that this only translates into a less than 3\% higher transmission distance for PMBPSK $(14,000 \mathrm{~km}$ for PM-BPSK versus $13,600 \mathrm{~km}$ for PS-QPSK). Additionally, PM-BPSK requires an approximately $56 \%$ longer FIR-filter for chromatic dispersion compensation since the number of taps scale with the square of the symbol-rate. For transmission over 170 spans this corresponds to 1498 taps as opposed to 3371 taps [11], which translate into 2048 and 4096 taps when ceiled to the nearest power of two for implementation with the 
overlap and save technique. Furthermore, the lower symbol-rate of PS-QPSK leads to 33\% lower electrical bandwidth requirements for transmitter and receiver-side electronics compared to PM-BPSK.

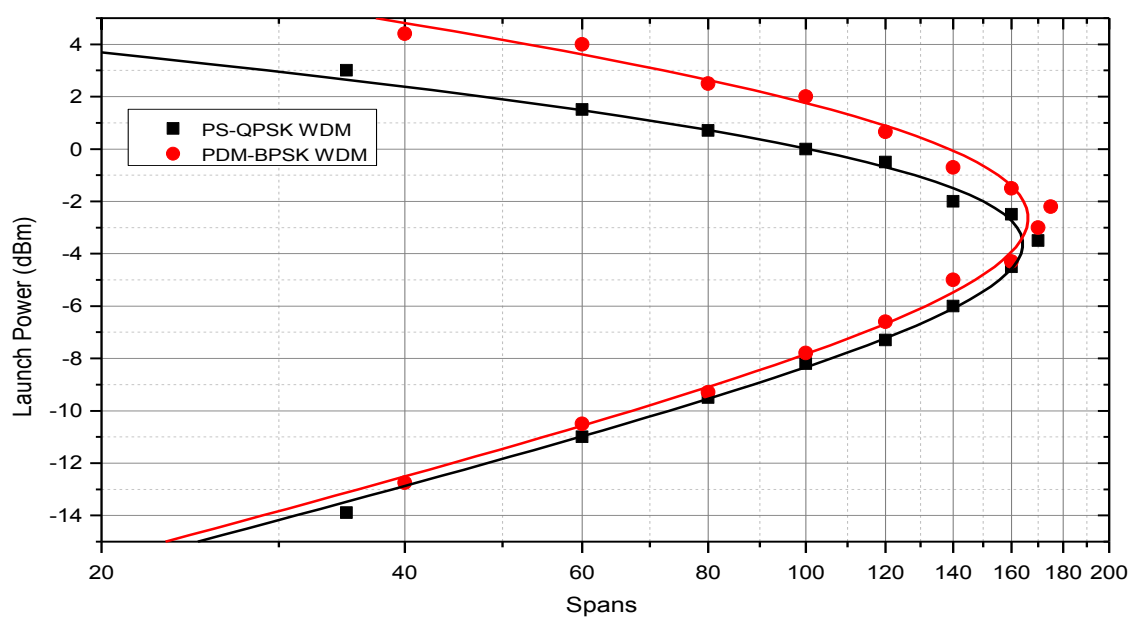

Figure 3: Achievable transmission distance of 42.9Gbit/s PM-BPSK and PS-QPSK at BER=3.8 $\times 10^{-3} .7 \mathrm{WDM}$ channels were transmitted on a $50 \mathrm{GHz}$ grid achieving maximum transmission distances in excess of $13,600 \mathrm{~km}$ with a span-length of $80 \mathrm{~km}$.

\section{Conclusions}

We compared, for the first time, ultra-long haul transmission of PS-QPSK and PM-BPSK at 42.9Gbit/s. WDMtransmission over an uncompensated SMF link employing 80km spans with EDFA-only amplification and phase and polarization-diverse coherent detection was investigated experimentally. Due to its reduced resilience to nonlinearities, PS-QPSK yielded a marginally lower transmission distance of $13,600 \mathrm{~km}$ as opposed to $14,000 \mathrm{~km}$ for PM-BPSK. However, due to its reduced symbol-rate PS-QPSK offers the benefits of reduced receiver complexity and lower bandwidth requirements for transmitter and receiver electronics which makes it attractive for ultra-long haul transmission.

\section{Acknowledgements}

The work described in this paper was carried out with the support of Huawei Technologies, the BONE-project, EPSRC, Oclaro, Yokogawa Electric Corporation and The Royal Society.

\section{References}

[1] G. Charlet et al., "Transmission of 81 channels at 40Gbit/s over a Transpacific-Distance Erbium-only Link, using PDM-BPSK Modulation, Coherent Detection, and a new large effective area fibre.", Proc. ECOC 2008, Paper Th.3.E.3, Sept. 2008.

[2] D. Foursa et al., "Coherent 40Gb/s Transmission with High Spectral Efficiency Over Transpacific Distance", Proc. OFC/NFOEC 2011, Paper OMI4, Mar. 2011.

[3] M. Karlsson and E. Agrell, "Which is the most power-efficient modulation format in optical links?", Opt. Express, vol. 17, no. 13, pp. 1081410819, Jun. 2009.

[4] E. Agrell and M. Karlsson, "Power-Efficient Modulation Formats in Coherent Transmission Systems", J. of Lightw. Technol., vol. 27, no. 22, pp. 5115-5126, Nov. 2009.

[5] J. Gaudette et al., “40Gb/s and 100Gb/s Ultra Long Haul Submarine Systems”, Proc. SubOptic 2010, Paper THU-1C-3, May 2010

[6] S. Makovejs et al., "Characterisation of long-haul 112Gbit/s PDM-QAM-16 transmission with and without digital nonlinearity compensation”, Opt. Express, vol. 18, no. 12, pp. 12939-12947, Jun. 2010.

[7] D. S. Millar et al., "Generation and long-haul transmission of polarization-switched QPSK at $42.9 \mathrm{~Gb} / \mathrm{s}$ " Accepted for publication in Opt. Express.

[8] D. S. Millar and S. J. Savory, "Blind Adaptive Equalization of Polarization Switched QPSK Modulation", Accepted for publication in Opt. Express

[9] S. J. Savory, G. Gavioli, R. I. Killey and P. Bayvel, "Electronic compensation of chromatic dispersion using a digital coherent receiver", Opt. Express, vol. 15, no. 5, pp.2120-2126, Mar. 2007.

[10] S. J. Savory et al., "Impact of Interchannel Nonlinearities on a Split-Step Intrachannel Nonlinear Equalizer", Photon. Tech. Lett., vol. 22, no. 10, pp. $673-675$, May 2010.

[11] S.J. Savory, “Digital Filters for Coherent Optical Receivers”, Opt. Express, vol. 16, No. 2, pp. 804-817, Jan. 2008. 